\title{
Corn Snakes Can Behaviorally Enhance Crypsis by Choosing Complex Backgrounds and Substrate
}

\author{
Lindsay Kravchuk ${ }^{1}$ and Charles M. Watson ${ }^{*}, 1,2$
}

\begin{abstract}
${ }^{1}$ McNeese State University, Department of Biology, Louisiana, USA.
${ }^{2}$ Midwestern State University, Department of Biology, Texas, USA

*Corresponding author (Email: charles.watson@msutexas.edu)
\end{abstract}

Citation - Kravchuck, L., \& Watson, C. M. (2020). Corn snakes can behaviorally enhance crypsis by choosing complex backgrounds and substrate. Animal Behavior and Cognition, 7(1), 39-48. https://doi.org/10.26451/abc.07.01.04.2020

\begin{abstract}
Many organisms have evolved coloration that increases their likelihood of survival. Crypsis is one such mechanism employed by many animals to avoid detection by blending into their habitat. By remaining unseen, these animals can enhance predation avoidance and prey acquisition. If an animal can recognize backgrounds and substrates that more closely match its own color and pattern within its habitat, then it could behaviorally enhance the effectiveness of crypsis and therefore further increase its own fitness. We use a common cryptic reptile, the corn snake (Pantherophis gutattus), to test whether snakes can recognize complexity and shade of their habitat and then position their body in substrates or on backgrounds that maximize concealment as perceived by a human. We use equal-options choice trials to determine two-dimensional background choice and pairwise comparisons to determine the hierarchy of choice among three-dimensional substrates. We show that corn snakes tend to choose more complex backgrounds and substrates while avoiding the high contrast of solid-white backgrounds. This indicates that these organisms seek out areas where their cryptic color and pattern are most effective.
\end{abstract}

Keywords - Crypsis, Background, Substrate, Choice

Predator-prey interactions exert selective pressures to make predators more effective at capture and prey more effective at avoiding capture. One outcome of this evolutionary arms race is adaptive coloration, or changes in color and/or pattern that serve as mechanisms to avoid predation or increase the likelihood of capturing prey (Cott, 1940). This includes such strategies as decoy coloration (Watson et al., 2012; Wilkinson, 2003), flash coloration (Bae et al., 2019; Murali, 2018), motion-dazzle coloration (Murali \& Kodandaramaiah, 2017; Stevens et al., 2011), aposematism (Mappes et al., 2005; Rojas et al., 2015), masquerade (Allen \& Cooper, 1985; Skelhorn et al., 2010), and crypsis (Ruxton et al., 2004). Crypsis allows organisms to avoid detection by predators and prey by exhibiting coloration and/or patterns that make them less conspicuous to would-be predators and prey (Edmunds, 1974). In order to be cryptic, Endler (1978) suggests that the color and pattern of the organism must approximate the same grain, color frequency or diversity, and brightness distribution/contrast as the background. Some cryptic organisms almost completely disappear into the background while others appear only minimally hidden, at least to the human eye. The effectiveness of cryptic coloration depends upon the ability of the organism's prey or predators to perceive small differences between the organism and the background (Cott, 1940; Cronin, 2005; Stevens \& Cuthill, 2006). 
There are multiple strategies under the general umbrella of crypsis. Many organisms are colored and patterned so that they match the background in their habitat (Cott, 1940; Endler, 1978; Merilaita et al., 2017; Thayer, 1918). Other examples of crypsis that are not directly associated with background matching include patterns to break up and conceal the edge and shape of an organism (Stevens \& Cuthill, 2006) or "masquerading" as inedible or inanimate objects naturally found in the habitat, such as feces, dry leaves, or rocks (Allen \& Cooper, 1985; Skelhorn et al., 2010). If the background is not sufficiently complex to allow the animal's edge to be broken up or concealed or is so homogeneous that any object would stand out, these strategies may be rendered ineffective. The masquerade strategy may still confer an advantage to the animal in such scenarios if would-be predators and potential prey still misidentify the animal as inedible or inanimate (Skelhorn et al., 2010).

There are many examples of organisms evolving crypsis to blend in with complex structures such as lichens and moss (Bonte \& Maelfait, 2004; Kikuchi et al., 2017). There are also instances of local coloration adaptation to monochromatic, simple backgrounds in lizards (Rosenblum et al., 2010) and mice (Hoekstra et al., 2004; Sumner, 1921) occurring in desert regions, where they exhibit blanching in homogeneously pale sandy conditions and melanism when living on dark igneous rocks. Thus, crypsis can evolve along a continuum ranging from a complex mosaic of color and pattern to a patternless, monochromatic phenotype based primarily upon the color and pattern of the background.

Cryptic strategies also range from mostly passive, whereby the organism evolves to match the habitat with a static color and pattern (Cuthill et al., 2005; Farallo \& Forstner, 2012; Merilaita et al., 2017), to active, whereby the organism actually perceives the background and undergoes physical and biochemical changes to match it (Stuart-Fox \& Moussalli, 2009). Among the many groups of animals that exhibit such color change are frogs (King et al., 1994; Stegen et al., 2004), fish (Donnelly \& Whoriskey Jr, 1991; Kelman et al., 2006), lizards (Macedonia, 2001; Stuart-Fox et al., 2006), and even crocodilians (Merchant et al., 2018). Extreme examples of color and pattern change include octopi and cuttlefish that recognize their environment and change the color and texture of their body to closely match the background (see Hanlon, 2007; Josef et al., 2012; Kelman et al., 2008). Through background and substrate choice, those species that cannot change color and pattern quickly may also be able to enhance the effectiveness of their cryptic phenotype by choosing those places within their habitat where it is most effective. If the organism is cognitively and behaviorally able to match its own color and pattern to similarly colored and patterned backgrounds, then it could therefore increase the effectiveness of its own adaptive coloration and thereby further increase its fitness (Stevens \& Ruxton, 2019). Snakes represent a group of organisms that are generally unable to rapidly change color or pattern, yet they occupy a broad range of habitats. Many snake species, including some of the smallest and largest, rely on crypsis to avoid detection (Greene, 2000). Some snakes are also ambush predators that rely heavily on crypsis to obtain food (Greene, 2000; Zug et al., 2001).

Although snakes have cones and rods and many squamates do have color vision, there is no behavioral evidence to date that suggests that snakes generally perceive color (Simões et al., 2016). The corn snake (Pantherophis guttatus Linnaeus, 1776), of the southeastern United States is a medium to large-sized (76 - $122 \mathrm{~cm}$ total length) snake that inhabits a broad range of habitats, including grasslands, forests, and rocky outcroppings. It is also adept at climbing trees and can be found in subterranean rodent burrows (Conant \& Collins, 1998; Gibbons, 2017). Its coloration ranges from bright orange, brown, to gray with red to brown blotches along the length of its body and tail (Conant \& Collins, 1998). As a habitat generalist and active forager, corn snakes routinely encounter a variety of colors and textures on which and in which it travels. Sharp-eyed predators, such as hawks, would be better able to identify and attack snakes that stand out against their backdrop. This species is particularly well suited for laboratory trials because it is routinely bred for the pet trade and its husbandry is well established (Love \& Love, 2006; Mitchell, 2004). Therefore, specimens are readily available, inexpensive, and easy to maintain in the laboratory. Here we test whether this common cryptic snake can recognize differences in shades and pattern and choose backgrounds and substrates that may make it more difficult to detect by predators and prey. 


\section{Methods}

\section{Study Specimens}

The specimens tested in this study were naïve hatchlings (1-3 weeks post-hatch) that were wary of humans and exhibited fear behavior, such as quickly moving away, striking, tucking the head under the body, and aggressive bluffing (Keogh \& DeSerto, 1994), when unable to hide. All the specimens used were generally orange and gray-brown non-siblings obtained from a professional breeder of corn snakes. This ensured that the snakes had all experienced the same post-hatch regime, were of the same age/size ( \pm 2 weeks age range/18-22 cm size range) and were unrelated. As common practice among snake breeders, they were sold after initial ecdysis and first feeding. Snakes were handled minimally over the duration of the experiment to avoid habituation to human contact. The design of this experiment required that the snakes be fearful of humans and prefer to remain concealed. This increased the likelihood that, if they could, they would choose backgrounds that help them blend in and avoid detection.

Over the course of this study, snakes were housed in plastic containers $(17 \mathrm{~cm} \mathrm{x} 43 \mathrm{~cm}$ x $8 \mathrm{~cm})$ and fed size-appropriate, previously frozen rodents weekly. They were kept on a substrate of shredded aspen and provided water ad libitum. Animals were kept on a $12 \mathrm{~h} / 12 \mathrm{~h}$ light regime with a minimally fluctuating ambient temperature of $26^{\circ} \mathrm{C}$. Lighting was provided by overhead fluorescent bulbs used to light the laboratory. All procedures followed McNeese State University's policies and procedures at the time this research was performed and comply with all U.S. regulatory agency policies and guidelines (Council, 2010; NIH, 1986). The same 25 snakes participated in each trial.

\section{Background and Substrate Colors and Patterns}

Four colors and patterns were used to test background and substrate choice (Figure 1). Solid white was used to represent a patternless light background and solid black was used to represent a patternless dark background. Black and white represents the extremes of light and dark monochromatic substrates. A checkered pattern consisting of $0.5 \mathrm{~cm}$ black and white squares represents a complex pattern that does not closely mimic the snake's own color and pattern and, therefore, poorly conceals it. The final pattern was made by taking high-resolution digital images of the snakes and merging them to create a single repetitive pattern that replicates the size, color and pattern of the specimens used at a 1:1 scale. This color and pattern most effectively conceals the snake to human visual perception. Over the course of a snake's daily activity, it will encounter situations where it must travel on top of impenetrable substrate (i.e., bare patches of the forest floor, rock, logs, or compact leaf litter), and substrates in which it can partially or completely immerse its body (i.e., loose leaf litter, sloughed bark, or tall grass). To consider both of these situations, we devised two separate, but related experiments.

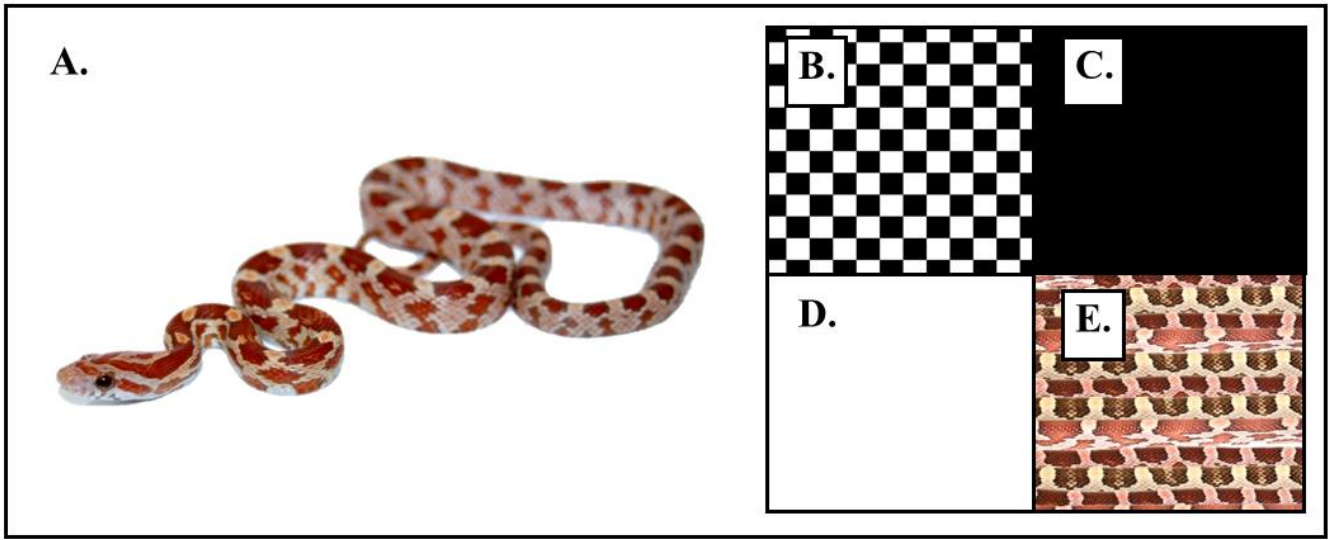

Figure 1. A corn snake (A.) and background colors and patterns (B. black and white checkered, C. solid black, D. solid white, and E. snake color and pattern) used in this study. 


\section{Experiment 1: Background Choice}

For the purpose of this study we define background as a two-dimensional plane that the snake can sit on top of, but not hide in or under. This tests the snake's ability to recognize shade and pattern without the confounding effects of physical concealment. We tested two-dimensional substrate preference using a $24 \mathrm{~cm}$ diameter disk $\left(452.4 \mathrm{~cm}^{2}\right)$ with equal-sized quadrants $\left(113.1 \mathrm{~cm}^{2}\right)$ for each background pattern. This paper disk was laminated for ease of cleaning and placed in the bottom of a standard white $\sim 19 \mathrm{~L}$ bucket. The white and black treatments were opposite one another as was the checkered and snakepattern. Snakes were placed in the middle of the container with no regard for the position of the head. Therefore, placement was functionally random. Each snake was left in the container for 20 min, at which time the background containing most of the snake's body was noted. The duration of 20 min was determined through preliminary trials to allow the snakes a brief acclimation period during which they explored the container before settling into a single spot, usually coiled. If left for more than 20 min, snakes in the preliminary trial often began exploring the container again, presumably because they felt the threat has passed. This was consistent among repeated preliminary trials using three juvenile corn snakes and two black rat snakes (Pantherophis obsoletus, a closely-related congener). No snake used in preliminary trials was used in the experimental trials. After each experimental trial, the laminated backgrounds were cleaned with a 50\% Isopropyl Alcohol solution, rinsed, washed with dishwashing soap, and rinsed again to ensure no chemical cues were left from the previous snake. This was performed once per snake $(n=25)$ using five identical arenas. A chi-square test was used to determine if the snakes' choice deviated significantly from random $(\alpha=.05)$. All trials for both experiments were performed a minimum of one week, but no more than two weeks, after the snakes' last meal.

\section{Experiment 2: Substrate Choice}

Hiding in or under loose substrate, such as leaf litter or vegetation is another way that snakes may avoid detection. In these situations, parts of the snakes may remain exposed or partially visible between elements of the substrate. Because habitats are three-dimensional, choice of such substrates may be more relevant to the real world than flat background matching. We produced four different substrates by printing double-sided copies of the four colors and patterns and shredded them using a standard strip paper shredder into $\sim 1.25 \mathrm{~cm}$ wide strips. The white and black substrate were made by simply shredding white and black paper. The shredded substrate was then fluffed and placed in the bottom of an $85 \mathrm{~L}$ sweater box (base: $76.2 \mathrm{~cm}$ x $48.6 \mathrm{~cm}$ ) so that the bottom was covered by $\sim 5 \mathrm{~cm}$ of substrate. Each trial consisted of two substrates, each covering half of the container floor. All possible pairwise comparisons were tested for each of the 25 snakes to produce a hierarchy of choice. The snakes were placed in the center of the container with no regard for position of the head, decreasing the likelihood of placement bias. The order in which we presented each snake to pairwise tests of substrate choice was random. Each snake was left in the container for $20 \mathrm{~min}$, after which time the position of the snake was noted and the trial ended. Each snake was used only once for each pairwise trial. After each trial, the substrate was removed and discarded and the inside of the enclosure was cleaned using the same procedure described earlier for background choice. In most cases, the snake's body was completely covered by one substrate or the other with the snake still detectable between strips of substrate. In the rare instance that their body spanned the two substrates $(n=2)$, the position of the head and the majority of the body was marked as the preferred substrate.

These pairwise choices were evaluated using a chi-square test with equal choice as the expected outcome $(\alpha=.05)$. We then used a procedure adapted from Saaty's Analytic Hierarchy Process (HAP; Saaty, 1980, 2008) in which we constructed a choice matrix based upon the results of the chi-square test. Since our data are binomial (preferred or not preferred), the typical nine-point scale of preference used in HAP is not applicable. Our simplified methodology allows us to conservatively rank and evaluate binomial data from pairwise choice trials using results from the statistical tests. If the pairwise trial was statistically significant, then the chosen substrate was assigned the entire matrix cell. If choice was 
statistically indistinguishable from a pairwise comparison, both substrates were assigned to the cell. Therefore, each substrate was awarded a value of $0,0.5$ or 1 for each cell. The sum value from each substrate choice was then divided by the total number of cells in the matrix. Using these weighted values, we ranked the substrate choices. For both experiments, a single observer (LK) recorded all data.

\section{Results}

\section{Experiment 1: Background Choice}

The snakes exhibited a significant preference for one of the complex colors and patterns and an avoidance of one of the patternless monochromatic backgrounds $\left(X^{2}(3, N=25)=8.44, p=.038\right)$. The white background was selected significantly less often than expected and the checkered pattern was selected at a higher frequency than expected under an equal choice scenario. The black and snake-colored backgrounds were selected near the expected frequency of 6.25 (Figure 2).

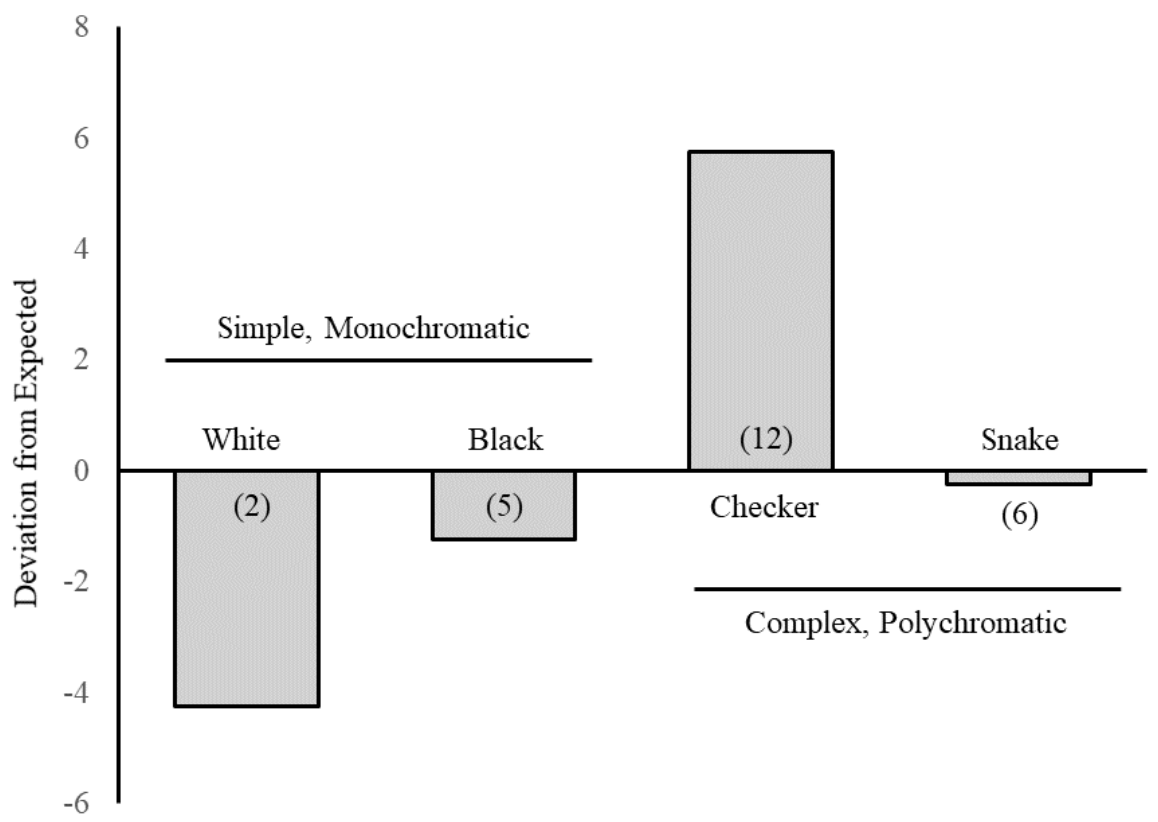

Figure 2. Deviation from expected background choice frequency by the corn snake (expected $=6.25$ for each, observed frequency in parentheses) for the four color/patterns tested. $\left(X^{2}(3, N=25)=8.44, p=.038\right)$.

\section{Experiment 2: Substrate Choice}

Pairwise trials identified two-choice comparisons as significant (White $\mathrm{x}$ Checker and White $\mathrm{x}$ Snake Coloration, Tables $1 \&$ 2). Consistent with background choice, P. guttatus hatchlings chose complex patterns over solid colors, with both checkered and snake-colored substrates preferred equally, followed by solid black substrate. Solid white substrate was again chosen at the lowest frequency (Table 3). We regrettably failed to record the order of exposure to treatments and individual choice for each snake, which would have allowed us to account for and discuss preferences among and within individuals. These data would have been an interesting addition, but we argue that our findings nevertheless show clear choice and avoidance of different substrates over our relatively large sample. 
Table 1

Frequencies and Calculated Chi-square (Corrected for Continuity) Results for all Pairwise Comparisons of Substrate used in this Study.

\begin{tabular}{lcccc}
\hline Comparison Pair & Frequency & Chi-Square & $d f$ & $p$ \\
\hline White x Black & $11: 14$ & 0.16 & 1 & .690 \\
White x Checker* & $5: 20$ & 7.84 & 1 & .005 \\
White x Snake* & $4: 21$ & 10.24 & 1 & .001 \\
Black x Checker & $9: 16$ & 1.44 & 1 & .230 \\
Black x Snake & $12: 13$ & 0.00 & 1.000 \\
Checker x Snake & $17: 08$ & 2.56 & 1 & .110 \\
\hline
\end{tabular}

Note: *Significant Difference $(\mathrm{N}=25, \alpha=.05)$. Preferred substrate is bolded.

Table 2

Pairwise Choice of Substrate Matrix Constructed using Chi-square Results from Table 1.

\begin{tabular}{lcccc}
\hline & White & Black & Checker & Snake \\
\hline White & - & WB & C & S \\
Black & - & - & BC & BS \\
Checker & - & - & - & CS \\
Snake & - & - & - & - \\
\hline
\end{tabular}

Note: The beginning letter of each substrate represents that substrate in the matrix.

Table 3

Weight and Rank of Substrate Choice using the Pairwise Choice Matrix (Table 2).

\begin{tabular}{lccc}
\hline Substrate & \#Cells & Weight & Rank \\
\hline White & 0.5 & 0.08 & 3 \\
Black & 1.5 & 0.25 & 2 \\
Checker & 2.0 & 0.33 & 1 \\
Snake & 2.0 & 0.33 & 1 \\
\hline
\end{tabular}

\section{Discussion}

Based upon our knowledge of how crypsis works (Cott, 1940; Merilaita et al., 2017), we suppose snakes that rely on crypsis as an anti-predation mechanism experience higher rates of predation when they fail to adequately match their background. This predator-based selection can result in intraspecific color polymorphism that correlates with the predominant substrate color in the snake population's range (Farallo \& Forstner, 2012). One would expect snakes to choose those backgrounds or substrates that best conceal them from predators and prey. Isaac and Gregory (2013) confirmed this expectation, showing that garter snakes from two populations exhibiting distinct color differences tended to choose basking sites that best matched their own coloration. Conversely, Capula and Luiselli (1995) reported no difference in substrate preference between cryptic and melanistic forms of Vipera berus in the eastern Italian Alps, with both forms choosing dark backgrounds. In this case, the need for substrates with higher thermal 
quality (darker substrates) may override the cost of reduced crypsis in this relatively cold climate. These seemingly conflicting findings illustrate the need to consider other aspects of the organism's biology, such as physiology and behavior, when interpreting results of such experiments. It is also possible that snakes choose backgrounds or substrates for reasons independent of crypsis, such as to search preferred habitat of prey. It is with these considerations in mind that we interpret our findings.

If given a choice of these four backgrounds, corn snakes choose dark and complex backgrounds over plain white, which presumably leaves them most vulnerable to detection since they are most conspicuous to the human visual system against this background. White backgrounds would be comparable in nature to open expanses of monochromatic substrates such as pale sand, concrete, and limestone outcroppings. The snakes also selected the checkered background pattern at a greater frequency than any of the others, including the snake pattern (Figure 2). This may indicate that the snake recognizes the high contrast checkered pattern as complex and may not be able to discern the black background from the snake-colored background. Snakes generally exhibit less visual acuity than birds and mammals (Stuart-Fox et al., 2006) and may require greater contrast to consistently discern solid black from snake pattern and color. Nonetheless, this shows that snakes can minimally recognize simple differences in substrates and backgrounds and favor those that are complex rather than solid.

Based upon the pairwise choice trials, these snakes do not appear to discern between the complex pattern and the snake pattern. This is in contrast to the background choice study in which they showed negligible choice between black and snake-colored backgrounds. This may be attributable to the snake being able to better perceive the shades and pattern more clearly from the vantage point of immersion in the substrate. Because habitats are generally three-dimensional, we suspect that substrate choice is a better indication of the snake's ability to recognize complexity and orient its body to those substrates and backgrounds. The snakes did not discern between near-exact replicas of their color and pattern and a general complex pattern (black and white checkered), but in both experiments, we show that background choice is nonrandom in favor of complex patterns.

These findings highlight a behavioral component to crypsis in corn snakes whereby they may modify and enhance the effectiveness of their adaptive coloration. This parallels the empirical work of Sargent (1966) and Boardman et al. (1974) that found evidence of behavioral background matching in moths. In their work with killifish, Kjernsmo and Merilaita (2012) interestingly discovered that females of the species chose complex background patterns over those that best matched their own pattern. Our similar findings support the notion that background complexity rather than exact matching may play an important role in crypsis as an antipredation mechanism by increasing the amount of information that a predator must process while foraging and therefore confusing predators by approaching the limit of their processing capabilities (Merilaita, 2003). Our data fits this hypothesis, which offers an interesting potential explanation for why corn snakes chose visually complex (checkered) background over the background that most precisely matched their own color and pattern.

Whether our findings represent a true choice within complex patterns for the checkered pattern over the snake pattern or illustrates a lack of visual acuity by snakes to distinguish the snake pattern as complex, we find a clear avoidance of white, monochromatic substrate. The finding that these two experiments illustrate similar patterns of choice in both conditions is confirmatory if not directly comparative. By avoiding the substrate and background on which they are most noticeable, and choosing more complex substrates and backgrounds or darker substrates that better conceal them using the human visual system, corn snakes can reduce their chances of being detected by a predator or seen by potential prey. This would increase fitness by decreasing mortality and increasing nutritional resources (Merilaita et al., 1999). We therefore find evidence for an active component (behavioral background matching) that enhances the adaptive advantage underlying the evolution of the cryptic phenotype in the corn snake. By choosing more complex habitats, but not necessarily the habitats that best match themselves, snakes can behaviorally enhance their cryptic color and pattern that may have otherwise evolved in a passive, predator-mediated situation. Future studies with other snakes would gauge the universality of this phenomenon and help us better understand the variation in background and substrate matching in snakes and other reptiles. 


\section{Acknowledgements}

The authors thank Hannah Etheridge, Matthew Ferguson, Lucas LeDoux, Rebbekah Watson, and Mark Wygoda for their support over the course of this project. They also thank the Department of Biology and Health Sciences at McNeese State University, Department of Biology at Midwestern State University, and The Shearman Research Initiative Fund for supporting this research.

\section{References}

Allen, J. A., \& Cooper, J. M. (1985). Crypsis and masquerade. Journal of Biological Education, 19, $268-270$.

Bae, S., Kim, D., Sherratt, T. N., Caro, T., \& Kang, C. (2019). How size and conspicuousness affect the efficacy of flash coloration. Behavioral Ecology, 30, 697-702.

Boardman, M., Askew, R. R., \& Cook, L. M. (1974). Experiments on resting site selection by nocturnal moths. Journal of Zoology, 172, 343-355.

Bonte, D., \& Maelfait, J. P. (2004). Colour variation and crypsis in relation to habitat selection in the males of the crab spider Xysticus sabulosus (Hahn, 1832) (Araneae: Thomisidae). Belgian Journal of Zoology, 134, 3-7.

Capula, M., \& Luiselli, L. (1995). Is there a different preference in the choice of background colour between melanistic and cryptically coloured morphs of the adder, Vipera berus? Italian Journal of Zoology, 62, 253-256.

Conant, R., \& Collins, J. T. (1998). A field guide to reptiles \& amphibians: Eastern and central North America (Vol. 12). New York: Houghton Mifflin.

Cott, H. B. (1940). Adaptive coloration in animals. London: Methuen.

Council, N. R. (2010). Guide for the care and use of laboratory animals. Washington, D.C.: National Academies Press.

Cronin, T. W. (2005). The visual ecology of predator-prey interactions. In P. Barbosa, \& I. Castellanos (Eds.), Ecology of predator-prey interactions (pp. 105-138). Oxford UK: Oxford University Press.

Cuthill, I. C., Stevens, M., Sheppard, J., Maddocks, T., Párraga, C. A., \& Troscianko, T. S. (2005). Disruptive coloration and background pattern matching. Nature, 434, 72.

Donnelly, W. A., \& Whoriskey Jr, F. G. (1991). Background-color acclimation of brook trout for crypsis reduces risk of predation by hooded mergansers Lophodytes cucullatus. North American Journal of Fisheries Management, 11, 206-211.

Edmunds, M. (1974). Defence in animals: A survey of anti-predator defences. Longman Publishing Group.

Endler, J. A. (1978). A predator's view of animal color patterns. In M. K. Hecht, W. C. Steere, \& B. Williams (Eds.) Evolutionary biology (pp. 319-364). Boston MA: Springer.

Farallo, V. R., \& Forstner, M. R. (2012). Predation and the maintenance of color polymorphism in a habitat specialist squamate. PloS One, 7, e30316.

Gibbons, W. (2017). Snakes of the Eastern United States. Athens, GA: University of Georgia Press.

Greene, H. W. (2000). Snakes: The evolution of mystery in nature. Berkeley, CA: University of California Press.

Hanlon, R. (2007). Cephalopod dynamic camouflage. Current Biology, 17: R400-R404.

Hoekstra, H. E., Drumm, K. E., \& Nachman, M. W. (2004). Ecological genetics of adaptive color polymorphism in pocket mice: Geographic variation in selected and neutral genes. Evolution, 58, 1329-1341.

Isaac, L. A., \& Gregory, P. T. (2013). Can snakes hide in plain view? Chromatic and achromatic crypsis of two colour forms of the western terrestrial garter snake (Thamnophis elegans). Biological Journal of the Linnean Society, 108, 756-772.

Josef, N., Amodio, P., Fiorito, G., \& Shashar, N. (2012). Camouflaging in a complex environment-Octopuses use specific features of their surroundings for background matching. PloS one, 7, e37579.

Kelman, E. J., Osorio, D., \& Baddeley, R. J. (2008). A review of cuttlefish camouflage and object recognition and evidence for depth perception. Journal of Experimental Biology, 211, 1757-1763.

Kelman, E. J., Tiptus, P., \& Osorio, D. (2006). Juvenile plaice (Pleuronectes platessa) produce camouflage by flexibly combining two separate patterns. Journal of Experimental Biology, 209, 3288-3292.

Keogh, J. S., \& DeSerto, F. P. (1994). Temperature dependent defensive behavior in three species of North American colubrid snakes. Journal of Herpetology, 28, 258-261.

Kikuchi, D. W., Kattan, G. H., Murcia, C., \& Montealegre, F. (2017). Endless forms most hidden: Katydids that masquerade as moss. Ecology, 98, 2479. 
King, R. B., Hauff, S., \& Phillips, J. B. (1994). Physiological color change in the green tree frog: Responses to background brightness and temperature. Copeia, 422-432.

Kjernsmo, K., \& Merilaita, S. (2012). Background choice as an anti-predator strategy: The roles of background matching and visual complexity in the habitat choice of the least killifish. Proceedings of the Royal Society B: Biological Sciences, 279, 4192-4198.

Love, K., \& Love, B. (2006). Corn snakes: The comprehensive owner's guide. Mount Joy, PA: Companion House Books.

Macedonia, J. M. (2001). Habitat light, colour variation, and ultraviolet reflectance in the Grand Cayman anole, Anolis conspersus. Biological Journal of the Linnean Society, 73, 299-320.

Mappes, J., Marples, N., \& Endler, J. A. (2005). The complex business of survival by aposematism. Trends in Ecology \& Evolution, 20, 598-603.

Merchant, M., Hale, A., Brueggen, J., Harbsmeier, C., \& Adams, C. (2018). Crocodiles alter skin color in response to environmental color conditions. Scientific Reports, 8, 6174.

Merilaita, S. (2003). Visual background complexity facilitates the evolution of camouflage. Evolution, 57, 12481254.

Merilaita, S., Scott-Samuel, N. E., \& Cuthill, I. C. (2017). How camouflage works. Philosophical Transactions of the Royal Society B: Biological Sciences, 372, 20160341.

Merilaita, S., Tuomi, J., \& Jormalainen, V. (1999). Optimization of cryptic coloration in heterogeneous habitats. Biological Journal of the Linnean Society, 67, 151-161.

Mitchell, M. A. (2004). Snake care and husbandry. The veterinary clinics of North America: Exotic animal practice, $7,421-446$.

Murali, G. (2018). Now you see me, now you don't: Dynamic flash coloration as an antipredator strategy in motion. Animal Behaviour, 142, 207-220.

Murali, G., \& Kodandaramaiah, U. (2017). Body size and evolution of motion dazzle coloration in lizards. Behavioral Ecology, 29, 79-86.

National Institutes of Health (1986). Public Health Service policy on humane care and use of laboratory animals. Office for Protection from Research Risks, National Institutes of Health.

Rojas, B., Valkonen, J., \& Nokelainen, O. (2015). Aposematism. Current Biology, 25, R350-R351.

Rosenblum, E. B., Römpler, H., Schöneberg, T., \& Hoekstra, H. E. (2010). Molecular and functional basis of phenotypic convergence in white lizards at White Sands. Proceedings of the National Academy of Sciences, 107, 2113-2117.

Ruxton, G. D., Sherratt, T. N., \& Speed, M. P. (2004). Avoiding attack: The evolutionary ecology of crypsis, warning signals and mimicry. Oxford, UK: Oxford University Press.

Saaty, T. L. (1980). Analytic hierarchy process. Wiley Online Library.

Saaty, T. L. (2008). Decision making with the analytic hierarchy process. International Journal of Services Sciences, 1, 83-98.

Sargent, T. D. (1966). Background selections of geometrid and noctuid moths. Science, 154, 1674-1675.

Simões, B. F., Sampaio, F. L., Douglas, R. H., Kodandaramaiah, U., Casewell, N. R., Harrison, R. A., Hart, N. S., Partridge, J. C., Hunt, D. M., \& Gower, D. J. (2016). Visual pigments, ocular filters and the evolution of snake vision. Molecular Biology and Evolution, 33, 2483-2495.

Skelhorn, J., Rowland, H. M., Speed, M. P., \& Ruxton, G. D. (2010). Masquerade: Camouflage without crypsis. Science, 327, 51-51.

Stegen, J. C., Gienger, C. M., \& Sun, L. (2004). The control of color change in the Pacific tree frog, Hyla regilla. Canadian Journal of Zoology, 82, 889-896.

Stevens, M., \& Cuthill, I. C. (2006). Disruptive coloration, crypsis and edge detection in early visual processing. Proceedings of the Royal Society B: Biological Sciences, 273, 2141-2147.

Stevens, M., \& Ruxton, G. D. (2019). The key role of behaviour in animal camouflage. Biological Reviews, 94 , 116-134.

Stevens, M., Searle, W. T. L., Seymour, J. E., Marshall, K. L., \& Ruxton, G. D. (2011). Motion dazzle and camouflage as distinct anti-predator defenses. BMC Biology, 9, 81.

Stuart-Fox, D., \& Moussalli, A. (2009). Camouflage, communication and thermoregulation: Lessons from colour changing organisms. Philosophical Transactions of the Royal Society B: Biological Sciences, 364, 463470.

Sumner, F. B. (1921). Desert and lava-dwelling mice, and the problem of protective coloration in mammals. Journal of Mammalogy, 2, 75-86. 
Thayer, G. H. (1918). Concealing-coloration in the animal kingdom: An exposition of the laws of disguise through color and pattern; Being a summary of Abbott H. Thayer's discoveries. New York, NY: Macmillan Company.

Watson, C. M., Roelke, C. E., Pasichnyk, P. N., \& Cox, C. L. (2012). The fitness consequences of the autotomous blue tail in lizards: An empirical test of predator response using clay models. Zoology, 115, 339-344.

Wilkinson, M. H. (2003). Decoys in predation and parasitism. Comments on Theoretical Biology, 8, 321-338.

Zug, G. R., Vitt, L. J., \& Caldwell, J. P. (2001). Herpetology: An introductory biology of amphibians and reptiles. New York, NY: Elsevier. 